\title{
The influence of the procedure on the dynamic range of bleached silver halide emulsions
}

\author{
C. Neipp (1), A. Beléndez (1) and I. Pascual (2)
}

(1) Departamento de Física, Ingeniería de Sistemas y Teoría de la Señal. Universidad de Alicante. Apartado 99. E-03080 Alicante. SPAIN

(2) Departamento Interuniversitario de Óptica. Universidad de Alicante. Apartado 99. E-03080 Alicante. SPAIN

Corresponding author: A. Beléndez

Phone: $+34-6-5903651$

Fax: +34-6-5903464

E-mail: augusto@disc.ua.es 


\begin{abstract}
Bleached holograms are one of the most interesting techniques used to produce phase holograms of high quality on photographic emulsions. Of particular interest is the method of fixation-free rehalogenating bleaching. Using Kogelnik's Coupled Wave Theory we are able to obtain information about the refractive index modulation, the thickness and the absorption coefficient of the final holograms recorded in bleaching procedures. In this way, it is possible to analyze the particular mechanisms involved in the formation of the holograms. In this paper we are going to study the influence of the different experimental conditions on the refractive index modulation created inside the hologram. It will be demonstrated that it is possible to maintain the values of diffraction efficiency over a wide range of exposures if the correct experimental conditions are chosen. On the other hand, it will also be demonstrated that the use of fixation free rehalogenating bleaching techniques allows high refractive index modulations to be obtained. Moreover, it is possible to attain a theoretical diffraction efficiency of $100 \%$, which is only limited by absorption and scattering.
\end{abstract}




\section{1.- Introduction}

Bleached silver halide emulsions have long been used as a medium for recording volume phase holograms because they offer several attractive advantages [1]. These advantages include a relatively high sensitivity, improved chemical processing, the possibility of obtaining commercial films and the repeatability of the results. Volume holograms recorded in silver halide emulsions are commonly bleached to obtain phase holograms in order to increase diffraction efficiency. Unfortunately, although the resulting phase holograms have high diffraction efficiency, this is usually accompanied by an increase in the scattering of light from the silver halide grains [2] and a consequent reduction in image quality.

A particular bleaching method is the fixation-free rehalogenating bleach proposed by Hariharan [3], subsequently improved by Crespo et al [4] and Kostuk [5]. This technique has the advantage over bleaching with fixation that shrinkage of the emulsion [5] is minimized. Hariharan and Chidley [6] studied the influence of the potassium bromide concentration on the diffraction efficiency and scattering of the final hologram. In their research they show that the concentration of the potassium halide must be adjusted carefully in order to obtain high values of diffraction efficiency and low values of scattering.

Hariharan also studied the influence of the development step on the quality of the hologram [7]. He showed that the concentration of sodium sulfite influences the quality of the final hologram. This is due to the solvent action of the sodium sulfite. They also showed that the action of hardener developers has a counteractive effect on the index modulation created by the diffusion process, so that the diffraction efficiency is decreased when hardener developers are used. We will prove that the use of hardener developers does not prevent high refractive index modulations being achieved. In fact, better results are obtained when these developers are used, for example the $\mathrm{CW}-\mathrm{C} 2$ developer in the case of PFG-01 emulsions, which is known for creating a hardening action due to its oxidation products.

In order to determine the influence of the fixation free bleaching procedure on different emulsions, three emulsions were studied in these experiments: Agfa 8E75 HD, 
Slavich PFG-01 and BB-640. Although Agfa 8E75 HD plates were discontinued in 1997 it is interesting to use them in a comparative study with other emulsions because these plates are very well known. The three emulsions studied present some differences which must be taken into account in the experimental procedure and also in the analysis of the transmittance curves. There are differences in the thickness, $d$, refractive index, $n$, mean grain size of the silver halide grains suspended in the gelatin of the emulsion, $\delta$, mass per unit surface area of silver halide, $\mathrm{m}_{\mathrm{AgBr}}$ and concentration of silver halide grains, $\mathrm{N}$, etc. These differences affect the quality of the final holograms.

The concentration of silver halide grains inside the emulsion can be calculated using the following expression [8]:

$$
\mathrm{N}=\frac{6 \mathrm{~m}_{\mathrm{AgBr}}}{\mathrm{d} \pi \delta^{3} \rho}
$$

where $\rho$ is the density of silver bromide $\left(6470 \mathrm{~kg} / \mathrm{m}^{3}\right)$. The values of the thickness of the layer, $\mathrm{d}$, and the refractive index of the emulsion, $\mathrm{n}$, for Agfa 8E75 HD, BB-640 and PFG-01 emulsions were measured experimentally using a procedure based on the film resonance method reported by Tholl et al.[9], obtaining $d \sim 6 \mu \mathrm{m}$ and $n \sim 1.62$, for Agfa 8E75 HD emulsions, $\sim 7 \mu \mathrm{m}$ and $\mathrm{n} \sim 1.61$ for PFG-01 emulsions, and $\sim 8 \mu \mathrm{m}$ and $\mathrm{n} \sim$ 1.63 for BB-640 emulsions. The values of $\mathrm{m}_{\mathrm{AgBr}}$ and $\delta$ given by the manufacturer, and the values of $\mathrm{N}$ otained from equation (1) are illustrated in Table 1 for the three emulsions studied.

\section{2.- Experimental procedure}

The experiments were carried out on red sensitive BB-640, Slavich PFG-01 and Agfa 8E75 HD silver halide emulsions. Unslanted holographic transmission gratings were recorded using two collimated beams from a $15 \mathrm{~mW}$ He-Ne laser (633 nm), with the polarization vector perpendicular to the plane of incidence. The two beams, of equal intensity, impinged on the emulsion forming an angle of $45^{\circ}$ (in air). The separate beams impinged each at +22.5 and -22.5 to the normal. With the geometry described, the spatial frequency of the gratings was calculated as $\sim 1200$ lines $/ \mathrm{mm}$. 
After exposure, the plates underwent the processing procedure illustrated in Table 2, so that phase transmission holograms were finally obtained [10]. In order to study the influence of the development step, different developers were used after exposure (D-19, CW-C2, AAC, PAAP). Table 3 shows the composition of the developers. On the other hand, in order to study the influence of the bleach bath, the $\mathrm{B} / \mathrm{A}$ ratio (relation between the potassium bromide concentration and the oxidizer concentration) was varied taking the values: $2,4,8,15,30,60,120$. There are differences in the degree of hardening of the gelatin of the emulsions used in the experiments, and so the temperature of the bleach bath was changed depending on the emulsion used. Due to the softness of the gelatin of Slavich PFG-01 plates [11], the temperature of the bleach bath was maintained at $20 \mathrm{C}$. However, for Agfa 8E75 HD and BB-640 plates the temperature of the bleach bath was kept at $50 \mathrm{C}$ in order to soften the gelatin of the plates which is hardened to a higher degree than that of PFG-01 plates. On the other hand the temperature of the developers was kept at $20^{\circ} \mathrm{C}$ for all the plates. In the case of BB-640 plates a hypersensitization solution was also included before exposure in order to increase the sensitivity of the plates as well as to soften the gelatin of the emulsion [12].

The diffraction efficiency $\eta$ of the phase holograms recorded was obtained as the ratio of the measured diffracted beam intensity to the incident collimated probe-beam intensity of the He-Ne laser. In order to take into account Fresnel losses and absorption due to the glass substrate, this expression was corrected by multiplying by an appropriate factor. The losses were calculated using Fresnel equations for p-polarized light in order to take into account the reflections that take place at the different interfaces of the hologram.

The efficiency of the zero-order transmission $\tau$ was similarly obtained as the ratio of the measured transmitted beam intensity to the incident power and was corrected by the same factor.

\section{3.- Analysis of the diffraction efficiency and transmission efficiency}




\subsection{Expressions for diffraction efficiency and transmission efficiency of volume phase} transmission holograms

Firstly, we are going to analyze the theoretical background. Using Kogelnik's Coupled Wave Theory [13], an expression for both diffraction efficiency and transmission efficiency can be obtained.

The diffraction efficiency is given by [14]:

$$
\eta=\exp \left(-\alpha d / \cos \theta^{\prime}\right) \frac{\sin ^{2}\left(\gamma^{2}+\xi^{2}\right)^{/ 2}}{\left(1+\frac{\xi^{2}}{v^{2}}\right)}
$$

and the transmission efficiency:

$$
\tau=\exp \left(-\alpha d / \cos \theta^{\prime}\right)\left[1-\frac{\sin ^{2}\left(v^{2}+\xi^{2}\right)^{/ 2}}{\left(1+\frac{\xi^{2}}{v^{2}}\right)}\right]
$$

where for gratings with unslanted fringes, $v$ and $\xi$ are expressed as follows:

$$
v=\frac{\pi \Delta \mathrm{nd}}{\lambda \cos \theta^{\prime}}
$$

and:

$$
\xi=\frac{\pi d}{\Lambda \cos \theta}\left(\left|\sin \theta^{\prime}\right|-\frac{\lambda}{2 n \Lambda}\right)(5)
$$

$v$ controls the maximum achievable diffraction efficiency, $100 \%$ for $v=\pi / 2$ whereas $\xi$ indicates the deviation from the Bragg condition.

In equation (2) $\alpha$ takes into account the absorption (and also the scattering; we have no means of differentiating between the two at this point); $d$ is the thickness; $n$ is the mean refractive index; $\Delta \mathrm{n}$ is the index modulation; $\Lambda$ is the grating period; $\lambda$ is the wavelength of reconstruction in air: $\theta^{\prime}$ is the angle of reconstruction in the medium, which is related to the angle of reconstruction in air $\theta$ by Snell's law. For unslanted gratings the grating period $\Lambda$ is given by equation:

$$
\Lambda=\left(\lambda / 2 \sin \theta_{0}\right)
$$

where $2 \theta_{0}$ is the angle between the two beams in air. For gratings analyzed in this paper, 
$\theta_{0}=22.5$ and $\Lambda=0.827 \mu \mathrm{m}$.

Because the expression for diffraction efficiency obtained from equation (2) depends on the refractive index modulation, $\Delta \mathrm{n}$, thickness, $\mathrm{d}$, and absorption and scattering of the hologram, $\alpha$, we can obtain information about these parameters by fitting the theoretical function of diffraction efficiency to the experimental data of the angular response of the diffraction efficiency. However, the experimental measurement of $\tau$ as a function of the angle at reconstruction is easier and more precise, because the direction of the transmitted beam is not modified as the diffracted beam angle changes. Therefore we fitted the analytical expression for transmission efficiency, given by equation (3),to the experimental data of the angular response of the transmittance for the transmission diffraction gratings recorded in this study.

3.2 Influence of the product $\Delta n \cdot d$ on the dynamic range of the diffraction efficiency as a function of exposure.

As can be seen, using equation (4) the value of $v$ is determined by the product $\Delta \mathrm{n} \cdot \mathrm{d}$ of the refractive index modulation and thickness of the final hologram. For diffraction gratings recorded with a spatial frequency of $1200 \mathrm{lines} / \mathrm{mm}$, it is possible to calculate the range of values of $\Delta \mathrm{n} \cdot \mathrm{d}$ which yield a theoretical diffraction efficiency, $\eta_{\text {theor }}$, of over $80 \%$. From equation (2), if losses are not considered and if $v$ pertains to the range of values $1.107 \leq v \leq 2.034$, the diffraction efficiency remains over $80 \%$. If the calculation is done assuming that diffraction gratings of 1200 lines $/ \mathrm{mm}$ are recorded on the hologram, the values of $\Delta \mathrm{n} \cdot \mathrm{d}$ yielding these values of $v$ must remain in the interval $0.217 \mu \mathrm{m} \leq \Delta \mathrm{n} \cdot \mathrm{d} \leq$ $0.398 \mu \mathrm{m}$. Figure 1 (a) shows a typical curve of diffraction efficiency as a function of exposure. The product $\Delta \mathrm{n} \cdot \mathrm{d}$ is also represented (Figure $\mathrm{l}$ (b)) assuming a linear dependence on exposure. The shadowed zone corresponds to the interval $0.217 \mu \mathrm{m} s$ $\Delta \mathrm{n} \cdot \mathrm{d} \leq 0.398 \mu \mathrm{m}$, which means that the diffraction efficiency (if no losses are considered) remains over $80 \%$. As can be interpreted from the figures, in order for a high diffraction efficiency to be maintained over a wide range of exposures, it is necessary for the values of $\Delta \mathrm{n} \cdot \mathrm{d}$ to remain in the shadowed region in this interval of exposures. If the slope of the line increases (which could happen with an increase in the potassium bromide concentration), the dynamic range (range of exposures in which high diffraction efficiencies are obtained) diminishes. We will use these ideas to analyze the particular 
mechanisms involved in the formation of the hologram, together with the influence of the experimental conditions on the dynamic range.

Another interesting parameter that can be obtained is the value of $\Delta \mathrm{n} \cdot \mathrm{d}$ yielding the maximum diffraction efficiency at the Bragg angle $(\xi=0)$. This occurs when $v$ takes the value $\pi / 2$. In the case of the transmission holograms studied in this paper, when $v=$ $\pi / 2$, at the Bragg angle (in air) $\left(22.5^{\circ}\right)$ the product $\Delta \mathrm{n} \cdot \mathrm{d}$ takes the value of $\sim 0.31 \mu \mathrm{m}$, considering the refractive indexes of the emulsions studied: $\mathrm{n} \sim 1.61$ for Slavich PFG-01 plates, $\mathrm{n} \sim 1.62$ for Agfa 8E75 HD plates and, $\mathrm{n} \sim 1.63$ for BB-640 plates (these values of the refractive index were measured experimentally using a procedure based on the film resonance method reported by Tholl et al.[9]). The value of the thickness was found to be $\sim 7 \mu \mathrm{m}$ for Slavich PFG-01. This means that the highest value of diffraction efficiency is achieved with a high value of refractive index modulation $\Delta \mathrm{n} \sim 0.044$. In the case of Agfa $8 \mathrm{E} 75 \mathrm{HD}$ plates, $\mathrm{d} \sim 6 \mu \mathrm{m}$, the value of $\Delta \mathrm{n}$ needed is $\Delta \mathrm{n} \sim 0.05 \mathrm{l}$, and in the case of BB-640 plates, $d \sim 8 \mu \mathrm{m}$, a value of $\Delta \mathrm{n} \sim 0.038$ is needed to achieve the maximum theoretical diffraction efficiency. These are very high values of refractive index modulation, but we will show that rehalogenating bleaching techniques without a fixation step are efficient enough to create this refractive index modulation, using different developers, when Agfa 8E75 HD, BB-640 and PFG-01 plates are used to record bleached transmission holograms.

\section{4.- Experimental results}

\section{1.- Analysis of the influence of the bleaching step on the dynamic range}

In recent articles $[10,11,15]$ the influence of the bleach bath solution on the diffraction efficiency of phase transmission gratings was analyzed for the different emulsions studied. The bleach bath solution is composed of two different solutions: A and $\mathrm{B}$ (Table 4). The oxidizer is contained in solution A, whereas the potassium bromide is contained in solution $\mathrm{B}$. To obtain the bleach solution 1 part of $\mathrm{A}$ is mixed with 10 parts of distilled water and $X$ parts of $B$. The ratio $X=B / A$ indicates the relation between the potassium bromide concentration and the oxidizer concentration (potassium dichromate). The concentration of potassium bromide in the bleach bath determines the rate of the diffusion process, which takes place from the exposed to the unexposed zones. A high rate 
of the diffusion process favors the refractive index modulation. However, a large quantity of potassium bromide tends to increase the amount of light scattered by the final hologram. Therefore, it was necessary to adjust the values of the B/A ratio to obtain high diffraction efficiencies and low values of scattering. The conclusions of the experiments carried out were that the B/A ratio depended on the particular emulsion studied and on the developer used. We are now going to compare the response of the product $\Delta n \cdot d$ as a function of exposure for different $B / A$ ratios.

Firstly, we will analyze the response of the diffraction efficiency and the product $\Delta \mathrm{n} \cdot \mathrm{d}$ as a function of the exposure for diffraction gratings recorded on BB-640 plates developed with D-19 developer. Figure 2 shows the diffraction efficiency and the product $\Delta \mathrm{n} \cdot \mathrm{d}$ as a function of exposure for transmission diffraction gratings recorded on BB-640 emulsion and developed with D-19, for different values of the B/A ratio. As can be seen, as the $B / A$ ratio increases, the range of exposures over which the product $\Delta n \cdot d$ is maintained in the shadowed zone, corresponding to a diffraction efficiency (without considering losses) over $80 \%$, diminishes. This is because as the $\mathrm{B} / \mathrm{A}$ ratio increases the diffusion mechanism is favored. Therefore, the refractive index modulations that can be achieved are higher for higher $\mathrm{B} / \mathrm{A}$ ratios. As a consequence, the product $\Delta \mathrm{n} \cdot \mathrm{d}$ increases rapidly as a function of exposure, so the range of exposures corresponding to the shadowed zone becomes smaller.

\section{2.- Influence of the emulsion on the obtainable refractive index modulation}

In order to determine the storing possibilities of the different emulsions with the fixation-free rehalogenating bleaching technique, we recorded transmission holograms of 1200 lines/mm following the processing procedure in Table 1 using D-19 developer and $7 \mathrm{~B} / \mathrm{A}$ ratios: $2,4,8,15,30,60,120$.

Firstly, we will prove that independently of the emulsion, it is possible to obtain the necessary refractive index modulation, in other words, that which yields a theoretical diffraction efficiency of $100 \%$. As explained, the optimum value of the product $\Delta \mathrm{n} \cdot \mathrm{d}$ must be $\Delta \mathrm{n} \cdot \mathrm{d} \sim 0.31 \mu \mathrm{m}$ in order for the theoretical diffraction efficiency to be $100 \%$. Figure 3 shows the product $\Delta \mathrm{n} \cdot \mathrm{d}$ as a function of exposure for diffraction gratings recorded in the three emulsions studied with the different B/A ratios. On the one hand, it can be seen that the highest values of the product $\Delta \mathrm{n} \cdot \mathrm{d}$ were achieved when BB-640 and Agfa 8E75 HD plates were used to record the holograms. In fact, a value of $\Delta \mathrm{n} \cdot \mathrm{d}$ as high as $0.631 \mu \mathrm{m}$ was achieved when diffraction gratings were recorded on BB-640 emulsion 
and bleached with a B/A ratio of 60 , which corresponded to an exposure of 12650 $\mu \mathrm{J} / \mathrm{cm}^{2}$. On the other hand, it is also interesting to note that, with the exception of the holographic gratings recorded on PFG-01 emulsion bleached with B/A ratios of 2 and 4, the value of $\Delta \mathrm{n} \cdot \mathrm{d}=0.31 \mathrm{~mm}$ is surpassed for all the emulsions and B/A ratios considered. This fact implies that the method of fixation-free rehalogenating bleaching applied to the three emulsions studied is efficient enough to produce the necessary refractive index modulation. Therefore, the values of diffraction efficiency are only limited by the absorption and scattering that may be present in the final hologram.

Figure 4 shows the diffraction efficiency as a function of exposure for diffraction gratings recorded on Agfa 8E75, BB-640 and PFG-01 plates, which yielded the maximum diffraction efficiency. As can be seen, the best results were obtained for BB640 plates. Although it must be said that these high values of diffraction efficiency for BB-640 plates come at the cost of the sensitivity. Nonetheless, when BB-640 plates were used, the values of the diffraction efficiency remain over $75 \%$ for a range of exposures between 1000 and $10000 \mu \mathrm{J} / \mathrm{cm}^{2}$. In the case of Slavich PFG-01 plates, the values of diffraction efficiency remain over $60 \%$ for a range of exposures between 300 and $5000 \mu \mathrm{J} / \mathrm{cm}^{2}$. The explanation for the higher values of diffraction efficiencies obtained with BB-640 and Slavich PFG-01 plates as compared to those obtained with Agfa $8 \mathrm{E} 75 \mathrm{HD}$ plates is the high values of the absorption and scatter coefficient obtained when Agfa 8E75 HD plates were used for the recording of the holograms. The absorption and scatter coefficient was calculated as $1-\eta-\tau$, where $\eta$ is the diffraction efficiency and $\tau$ the transmission efficiency of the diffraction gratings. Figure 5 shows the absorption and scattering as a function of the exposure for bleached transmission diffraction gratings recorded on the three emulsions studied and bleached with the optimum B/A ratio. The values of absorption and scattering are over $15 \%$ in the case of holograms recorded on Agfa 8E75 HD plates, reaching as much as $94 \%$ for an exposure of $850 \mu \mathrm{J} / \mathrm{cm}^{2}$. The values of absorption and scattering corresponding to the PFG-01 plates are lower, varying between $8 \%$ and $27 \%$ for an exposure of $3660 \mu \mathrm{J} / \mathrm{cm}^{2}$. The lowest values of absorption and scattering correspond to the BB-640 plates. The maximum value of absorption and scattering, 18\%, was obtained for an exposure of 
$25600 \mu \mathrm{J} / \mathrm{cm}^{2}$, whereas the minimum value was as low as $0.5 \%$ for an exposure of 100 $\mu \mathrm{J} / \mathrm{cm}^{2}$. These differences in the absorption and scatter coefficient are the real cause of the low diffraction efficiencies obtained in the case of Agfa 8E75 HD plates.

\section{3.- Influence of the development on the diffusion mechanism}

In section 4.1 it was demonstrated that the dynamic range, that is the optimum exposure range, of the final diffraction gratings obtained in BB-640 emulsion is influenced by the $\mathrm{B} / \mathrm{A}$ ratio. As the $\mathrm{B} / \mathrm{A}$ ratio increases the dynamic range diminishes. In this section we will see that this is the general behavior observed when using different developers, although some differences arise depending on the developer used.

In this section we will study holographic transmission gratings recorded on Slavich PFG-01 plates developed with four different developers: D-19, AAC, PAAP, $\mathrm{CW}-\mathrm{C} 2$. There are some differences between these developers, which as will be seen influence the final characteristics of the holograms. For instance, CW-C2 and D-19 are known as tanning developers. The tanning action of $\mathrm{CW}-\mathrm{C} 2$ takes place during the development step, whereas the tanning action of the D-19 developer takes place during the bleaching bath due to the high content of sodium sulfite in the D-19 developer. The sodium sulfite inhibits the tanning action during the development step, but the oxidation products of this developer join to the gelatin of the emulsion and when put in contact with an oxidant (during the bleaching bath) the tanning action takes place. On the other hand, the oxidation products of AAC and PAAP developers are not tanning. These differences in the hardening properties of the individual developers will affect the diffusion process of silver ions during the bleaching bath. The higher the hardening of the gelatin, the more the diffusion process is limited. The refractive index modulation created using fixation-free rehalogenating bleaches is established from the unexposed to the exposed zones. But the differential degree of hardening of the gelatin, generated by tanning developers, creates a refractive index modulation from the exposed to the unexposed zones, that is, in the opposite direction. Thus, by using tanning developers, the refractive index modulation is reduced. 
Figure 6 shows the product $\Delta \mathrm{n} \cdot \mathrm{d}$ as a function of exposure for diffraction transmission gratings recorded on Slavich PFG-01 plates and developed with D-19, $\mathrm{AAC}$, PAAP and CW-C2. It can be seen, on the one hand, that the value of $\Delta \mathrm{n} \cdot \mathrm{d}=0.31$ $\mu \mathrm{m}$ is reached for diffraction gratings developed with PAAP and AAC for all the B/A ratios studied: $2,8,30$ and 120 . The maximum value of the product $\Delta \mathrm{n} \cdot \mathrm{d}$ is reached with the PAAP developer. In the range of exposures studied, it was $0.496 \mathrm{~mm}$ for an exposure of $440 \mu \mathrm{J} / \mathrm{cm}^{2}$ and a B/A ratio of 8 , whereas in the case of the AAC a value of $\Delta \mathrm{n} \cdot \mathrm{d}=0.559 \mathrm{~mm}$ was obtained for an exposure of $440 \mu \mathrm{J} / \mathrm{cm}^{2}$ and a B/A ratio of 30 . Neither the PAAP nor the AAC developer creates a tanning action - there is no differential hardening which could oppose the diffusion process. For this reason, the values of the product $\Delta \mathrm{n} \cdot \mathrm{d}$ are only limited by the capability of the diffusion mechanism itself. In the case of D-19 and CW-C2 developers, there are B/A ratios for which the value of $\Delta n \cdot d=0.31$ is not reached in the range of exposures studied. In the case of diffraction gratings recorded with D-19, the optimum value of $\Delta n \cdot d$ is not reached for a B/A ratio of 2. The maximum value of $\Delta \mathrm{n} \cdot \mathrm{d}$ obtained is $0.249 \mu \mathrm{m}$ for an exposure of $1560 \mu \mathrm{J} / \mathrm{cm}^{2}$, which yields a theoretical diffraction efficiency of $91 \%$ (using equations (4) and (2)), although the diffraction efficiency obtained was $64 \%$ due to the absorption and scattering which was $24 \%$ in this case. For diffraction gratings developed with $\mathrm{CW}$ C2 developer, the optimum value of $\Delta \mathrm{n} \cdot \mathrm{d}$ is not reached when the holograms were bleached with a $B / A$ ratio of 30 . With this $B / A$ ratio the maximum value of $\Delta n \cdot d$ achieved was $0.284 \mu \mathrm{m}$ for an exposure of $1500 \mu \mathrm{J} / \mathrm{cm}^{2}$, which means a high theoretical diffraction efficiency of $99 \%$; although the diffraction efficiency measured was $67 \%$ due to a high value of the absorption and scatter coefficient - $32 \%$.

Another interesting matter arises when observing the $\Delta \mathrm{n} \cdot \mathrm{d}$ curves corresponding to the $\mathrm{CW}-\mathrm{C} 2$ developer. The lowest values of the product $\Delta \mathrm{n} \cdot \mathrm{d}$ are obtained with this developer. But the fact that with this developer the highest values of the product $\Delta \mathrm{n} \cdot \mathrm{d}$ are lower than those obtained with other developers does not prevent high diffraction efficiencies being attained. On the contrary, the result is a flatter response of diffraction efficiency as a function of exposure, as will be seen later. The reason for the lower values of the product $\Delta \mathrm{n} \cdot \mathrm{d}$ obtained with the $\mathrm{CW}-\mathrm{C} 2$ developer, as compared with the 
other developers, is the hardening character of the cathecol present in the $\mathrm{CW}-\mathrm{C} 2$ solution. This hardening effect opposes the diffusion mechanism, thereby limiting the refractive index modulation.

\section{4.- Diffraction efficiency and product $\Delta n \cdot d$ as a function of exposure}

Figures 7-10 show the diffraction efficiency and product $\Delta \mathrm{n} \cdot \mathrm{d}$ as a function of exposure for transmission diffraction gratings recorded on PFG-01 emulsion, developed with four different developers: D-19, CW-C2, PAAP and AAC and bleached with four different B/A ratios: $2,8,20$ and 120 . With the exception of the $\mathrm{CW}-\mathrm{C} 2$ developer, the same effects observed in section 4.1 can be seen. In other words, as the B/A ratio increases, the range of exposures where the theoretical diffraction efficiency remains over $80 \%$ diminishes.

Nevertheless, in the case of CW-C2 developer, this behavior is not seen. This is a consequence of the hardening effect created by this developer in the exposed zones, so that the diffusion process is limited. This fact is not a disadvantage, but actually permits a wider range of exposures in which the theoretical diffraction efficiency remains over $80 \%$. In Figure 10 it can be seen that, with the exception of plates bleached with a B/A ratio of 8 , the value of the product $\Delta \mathrm{n} \cdot \mathrm{d}$ is maintained in the range $0.217 \mu \mathrm{m} \leq \Delta \mathrm{n} \cdot \mathrm{d} \leq 0.398 \mu \mathrm{m}$, for a wide range of exposures, which explains the almost flat response of the diffraction efficiency. For instance, when the B/A ratio was 120 , the optimum range of exposures was between 125 and $3700 \mu \mathrm{J} / \mathrm{cm}^{2}$, with values of the product $\Delta \mathrm{n} \cdot \mathrm{d}$ of $0.252 \mu \mathrm{m}\left(\eta_{\text {theor }}=\right.$ $92 \%)$ and $0.271 \mathrm{~mm}\left(\eta_{\text {theor }}=97 \%\right)$, respectively. The maximum measured diffraction efficiency, $71 \%$, was achieved for an exposure of $290 \mu \mathrm{J} / \mathrm{cm}^{2}$ and corresponded to a value of the product $\Delta \mathrm{n} \cdot \mathrm{d}=0.276 \mu \mathrm{m}$, which yields a theoretical diffraction efficiency of $97 \%$, limited by a value of absorption and scattering of $28 \%$. An important conclusion can be made; the use of hardening developers in fixation-free rehalogenating techniques could be advantageous if flat responses of diffraction efficiency as a function of exposure are needed.

4.5.- Influence of the bleaching step on refractive index modulation and scattering of the final holograms

In this section we are going to present more evidence of the mechanism of hologram formation in the fixation-free rehalogenating techniques. As was explained by 
Hariharan, diffusion of silver ions from the exposed to the unexposed zones occurs during the bleaching bath. This diffusion increases the grain size of the silver halide grains in the unexposed zones in relation to their grain size in the exposed zones. Due to these differences in grain size, a refractive index modulation is created between the exposed and unexposed zones. As can be interpreted from the explanation for the mechanism of hologram formation, the increase in refractive index modulation will be accompanied by an increase in the scattering of the final hologram. Therefore, it is necessary to adjust the B/A ratio correctly in order to obtain high diffraction efficiencies and low values of scattering as explained in section 4.1.

In order to measure the scattering of the holograms recorded in this study, the experimental setup in figure 11 was used.

Figure 12 shows the refractive index modulation and scattering as a function of exposure for transmission diffraction gratings recorded on PFG-01 emulsion, developed with D-19 and bleached with 7 different B/A ratios: 2, 4, 8, 15, 30, 60, 120.

The interesting feature is that the evolution of the refractive index modulation as a function of exposure is similar to that of the scattering of the final hologram, thereby validating the hypothesis made about the hologram formation mechanism.

\section{5.- Conclusions}

By fitting the theoretical function of the transmittance to the experimental data of the angular response of the transmittance, information about the refractive index modulation, absorption and scatter coefficient and final thickness of the holograms can be obtained. We used this method to obtain information about the influence of the experimental conditions on the mechanism of hologram formation in fixation-free rehalogenating techniques. It was demonstrated that a flat response of the diffraction efficiency is influenced by the B/A ratio when D-19, AAC and PAAP developers were 
used. The dynamic range of exposures yielding high diffraction efficiencies decreases as the $\mathrm{B} / \mathrm{A}$ ratio increases.

On the other hand, it was demonstrated that the use of hardening developers, such as $\mathrm{CW}-\mathrm{C} 2$, permits widening of the dynamic range, since the hardening process limits the diffusion process, which is an advantage at high values of exposure.

More evidence is also given of the mechanism of hologram formation when analyzing the refractive index modulation and scattering curves as a function of exposure. It was observed that both the refractive index modulation and scattering of the holograms evolved with exposure in the same way, as predicted by the diffusion mechanism theory.

Finally it was demonstrated that the use of fixation free rehalogenating techniques with different emulsions and experimental conditions allows us to obtain the refractive index modulation necessary to yield a theoretical diffraction efficiency of $100 \%$, so that the diffraction efficiency is only limited by the absorption and scattering of the holograms.

\section{Acknowledgments}

This work was partially financed by the CICYT ("Comisión Interministerial de Ciencia y Tecnología", Spain) under project $n^{\circ}$ MAT2000-1361-C04-04 and the "Oficina de Ciencia y Tecnología" (Generalitat Valenciana, Spain) under project n GV01-130. 


\section{References}

[1] H. I. Bjelkhagen, Silver Halide Recording Materials for Holography and Their Processing (Springer-Verlag, Berlin, 1993).

[2] R. L. Van Renesse, "Scattering properties of fine-grained bleached emulsions", Photrgr. Sci. Eng. 24, 114-119 (1980).

[3] P. Hariharan, C.S. Ramanathan y G.S. Kaushik, "Simplified processing technique for photographic phase holograms", Optics Communications 3, 246-247 (1971).

[4] J. Crespo, A. Fimia, J.A. Quintana, Fixation-free methods in bleached reflection holography, Appl .Opt. 25, 1642-1645 (1986)

[5] R. K. Kostuk, "Factorial optimization of bleach constituents for silver halide holograms", Applied Optics 30, 1611-1616 (1991).

[6] P. Hariharan and C. M. Chidley, Photographic phase holograms: the influence of developer composition on scattering and diffraction efficiency, Appl. Opt. 26, 1230-1234 (1987).

[7] P. Hariharan, C.M. Chidley, Rehalogenating bleaches for photographic phase holograms: the influence of halide type and concentration on diffraction efficiency and scattering, Appl. Opt. 26, 3895-3898 (1987).

[8] N. J. Phillips, H. Heyworth y T. Hare, “On Lippmann's photography”, Journal of Photographic Science 32, 158-169 (1984). 
[9] H. D. Tholl, M. Döhmen y C.G. Stojanoff, "Determination of the mean refractive index and the thickness of dichromated gelatin holographic films using the thin film resonance method", Proceedings of SPIE 2405,76-87 (1996).

[10] C. Neipp, I. Pascual y A. Beléndez, "Optimization of a fixation-free rehalogenating bleach for BB-640 holographic emulsion", Journal of Modern Optics 47, 1671-1679 (2000).

[11] C. Neipp, E. Beronich, I. Pascual and A. Beléndez, "Fixation-free bleached silver halide transmission holograms recorded on Slavich PFG-01 red sensitive plates", Journal of Modern Optics 48, 1643-1655 (2001).

[12] A. Beléndez, C. Neipp, M. Flores and I. Pascual, "High-efficiency silver-halide sensitized gelatin holograms with low absorption and scatter", Journal of Modern Optics 45, 1985-1992 (1998).

[13] H. Kogelnik, "Coupled wave theory for thick hologram gratings", Bell Systems Technology Journal, 48, 2909-2947 (1969).

[14] C. Neipp, I. Pascual and A. Beléndez, "Silver halide sensitized gelatin derived from BB-640 holographic emulsion", Applied Optics 38, 1348-1356 (1999).

[15] C. Neipp, I. Pascual and A. Beléndez, "Bleached silver halide volume holograms recorded on Slavich PFG-01 emulsion: The influence of the developer", Journal of Modern Optics 48, 1479-1494 (2001). 


\section{Figure captions}

Figure 1.- (a) Diffraction efficiency versus exposure under the Bragg condition for theoretical transmission gratings. (b) product $\Delta n \cdot d$ versus exposure under the Bragg condition for theoretical transmission gratings.

Figure 2.- Diffraction efficiency and product $\Delta \mathrm{n} \cdot \mathrm{d}$ versus exposure under the Bragg condition for transmission gratings recorded on BB-640 emulsion developed with D-19 and bleached with 7 different B/A ratios: $2,4,8,15,30,60,120$.

Figure 3.- Product $\Delta \mathrm{n} \cdot \mathrm{d}$ versus exposure under the Bragg condition for transmission gratings recorded on three different emulsions, BB-640 Agfa 8E75 HD and Slavich PFG-01, developed with D-19 and bleached with 7 different B/A ratios: $2,4,8,15,30,60,120$.

Figure 4.- Diffraction efficiency as a function of exposure under the Bragg condition for transmission gratings recorded on three different emulsions, BB-640 Agfa 8E75 HD and Slavich PFG-01, developed with D-19, which yielded the maximum diffraction efficiency.

Figure 5.- Absorption and scattering as a function of exposure under the Bragg condition for transmission gratings recorded on three different emulsions, BB-640 Agfa 8E75 HD and Slavich PFG-01, developed with D-19, which yielded the maximum diffraction efficiency.

Figure 6.- Product $\Delta \mathrm{n} \cdot \mathrm{d}$ versus exposure under the Bragg condition for transmission gratings recorded on Slavich PFG-01 emulsion, developed with four different developers, D-19, CW-C2, AAC, PAAP and bleached with 4 different B/A ratios: $2,8,30,120$. 
Figure 7.- Diffraction efficiency and product $\Delta \mathrm{n} \cdot \mathrm{d}$ versus exposure under the Bragg condition for transmission gratings recorded on PFG-01 emulsion developed with D-19 and bleached with 4 different B/A ratios: 2, 8, 30, 120.

Figure 8.- Diffraction efficiency and product $\Delta \mathrm{n} \cdot \mathrm{d}$ versus exposure under the Bragg condition for transmission gratings recorded on PFG-01 emulsion developed with PAAP and bleached with 4 different B/A ratios: 2, 8, 30, 120.

Figure 9.- Diffraction efficiency and product $\Delta \mathrm{n} \cdot \mathrm{d}$ versus exposure under the Bragg condition for transmission gratings recorded on PFG-01 emulsion developed with $\mathrm{AAC}$ and bleached with 4 different $\mathrm{B} / \mathrm{A}$ ratios: 2, 8, 30, 120.

Figure 10.- Diffraction efficiency and product $\Delta \mathrm{n} \cdot \mathrm{d}$ versus exposure under the Bragg condition for transmission gratings recorded on PFG-01 emulsion developed with $\mathrm{CW}-\mathrm{C} 2$ and bleached with 4 different $\mathrm{B} / \mathrm{A}$ ratios: $2,8,30,120$.

Figure 11.- Experimental setup for the measurement of the scattering.

Figure 12.- Scattering and product $\Delta \mathrm{n} \cdot \mathrm{d}$ versus exposure under the Bragg condition for transmission gratings recorded on PFG-01 emulsion developed with D-19 and bleached with 7 different B/A ratios: 2, 4, 8, 15, 30, 60, 120. 
Table 1.- Values of mean grain size, $\delta$, mass of silver halide per unit surface $\mathrm{m}_{\mathrm{AgBr}}$ and concentration of silver halide grains, $\mathrm{N}$, for the three emulsions studied

\begin{tabular}{|l|ccc|}
\hline Emulsion & $\delta(\mathrm{nm})$ & $\mathrm{m}_{\mathrm{AgBr}}\left(\mathrm{g} / \mathrm{m}^{2}\right)$ & $\mathrm{N}\left(\right.$ grains $\left./ \mathrm{cm}^{3}\right)$ \\
\hline \hline Agfa 8E75 HD & 44 & 8.7 & $5.0 \times 10^{15}$ \\
BB-640 & 22 & 3.5 & $12.9 \times 10^{15}$ \\
& & & \\
PFG-01 & 40 & 2.7 & $1.7 \times 10^{15}$ \\
\hline \hline
\end{tabular}


Table 2.- Processing procedure

1.- Develop $5 \mathrm{~min}$

2.- Rinse in running water $1 \mathrm{~min}$

3.- Bleach for $1 \mathrm{~min}$ after the plate has cleared

4.- Rinse in running water $5 \mathrm{~min}$

5.- Dry at room temperature 
Table 3.- Composition of the developers

\begin{tabular}{ll}
\multicolumn{1}{c}{ D-19 } & \\
Metol & $\mathrm{gg}$ \\
Sodium sulfite & $45 \mathrm{~g}$ \\
Hydroquinone & $8 \mathrm{~g}$ \\
Sodium carbonate (anhydrous) & $50 \mathrm{~g}$ \\
Potassium bromide & $5 \mathrm{~g}$ \\
$\begin{array}{l}\text { Distilled water } \\
\quad\left(5 \text { minutes at } 20^{\circ} \mathrm{C}\right)\end{array}$ & 1 liter
\end{tabular}

\section{$\mathrm{AAC}$}

Ascorbic acid-L $\quad 18 \mathrm{~g}$

Sodium carbonate (anhydrous) $\quad 60 \mathrm{~g}$

Distilled water 1 liter

$\left(4\right.$ minutes at $\left.20^{\circ} \mathrm{C}\right)$

Two solutions, one containing the sodium carbonate and the other containing the ascorbic acid, are mixed just before use to form the final solution,

\section{$\mathrm{CW}-\mathrm{C} 2$}

Catechol

Ascorbic acid-L

Sodium sulfite (anhydrous)

Urea

Sodium carbonate

Distilled water
$10 \mathrm{~g}$

$5 \mathrm{~g}$

$5 \mathrm{~g}$

$50 \mathrm{~g}$

$30 \mathrm{~g}$

1 liter

Two solutions, one containing the sodium carbonate and the other containing the ascorbic acid and catechol, are mixed just before use to form the final solution.

\section{PAAP}

$\begin{array}{ll}\text { Phenydone } & 0.5 \mathrm{~g} \\ \text { Ascorbic acid-L } & 18 \mathrm{~g} \\ \text { Sodium hydroxide } & 12 \mathrm{~g} \\ \text { Sodium phosphate } & 28.4 \mathrm{~g} \\ \text { Distilled water } & 1 \text { liter }\end{array}$

$\left(4\right.$ minutes at $20^{\circ} \mathrm{C}$ ) 
Table 4.- $\quad$ Bleach bath composition (modified version of R-10)

\begin{tabular}{ll}
\multicolumn{2}{c}{ Solution $A$} \\
Potassium dichromate20 $\mathrm{g}$ \\
Sulfuric acid & $15 \mathrm{ml}$ \\
distilled water & 11 \\
$\qquad$ \\
Solution $B$ \\
potassium bromide & $100 \mathrm{~g}$ \\
distilled water & 11
\end{tabular}

Just before use, mix 1 part of $A$ with 10 parts of distilled water and add X parts of $B-B / A=X$. 

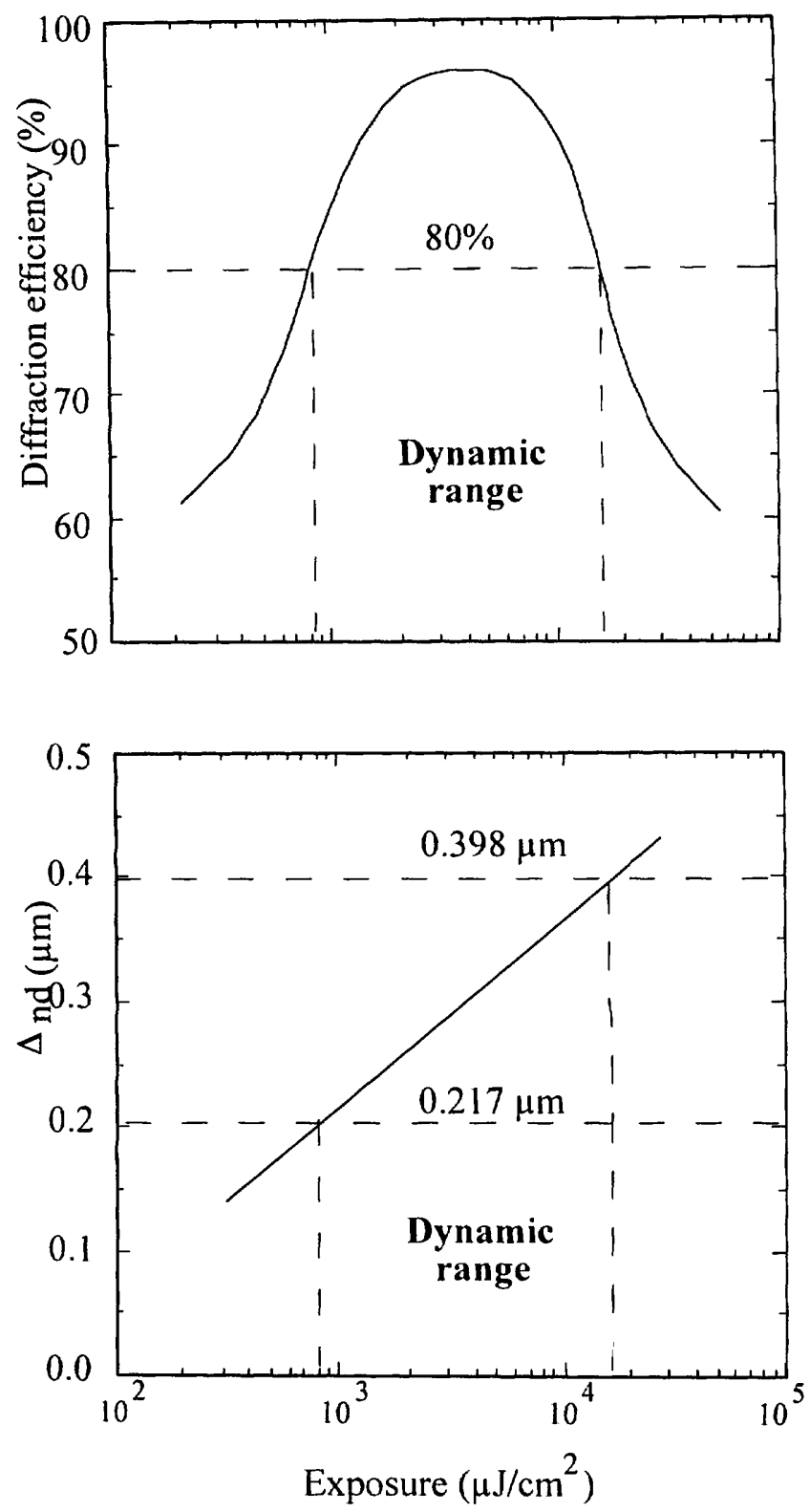

FIGURE 1 

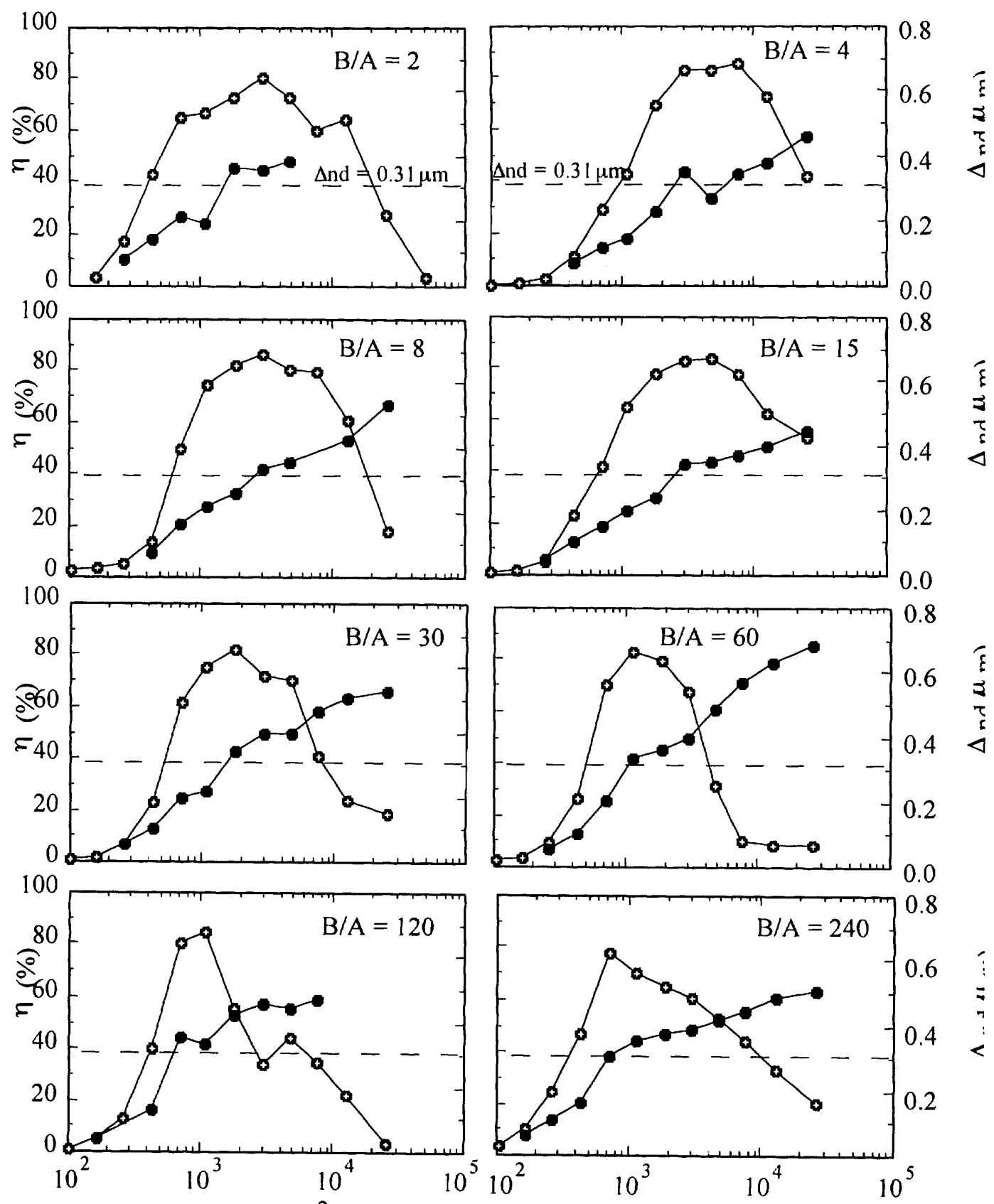

$\mathrm{E}\left(\mu \mathrm{J} / \mathrm{cm}^{2}\right)$

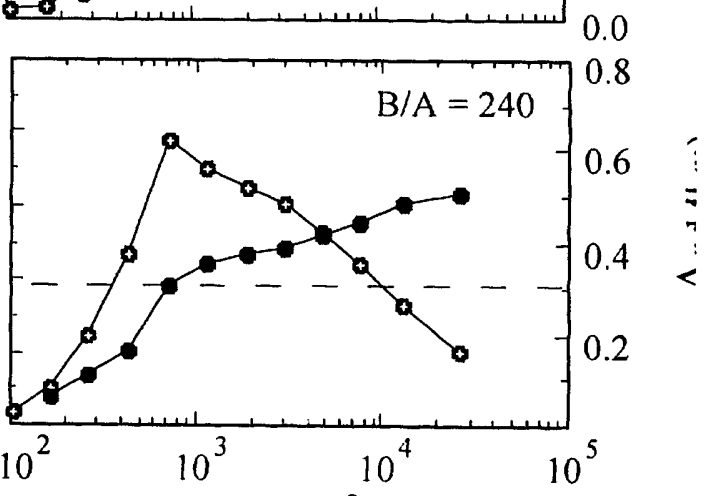

$\begin{array}{ll}\longrightarrow & \eta(\%) \\ \longrightarrow & \Delta \mathrm{nd}(\mu \mathrm{m})\end{array}$

FIGURE 2 

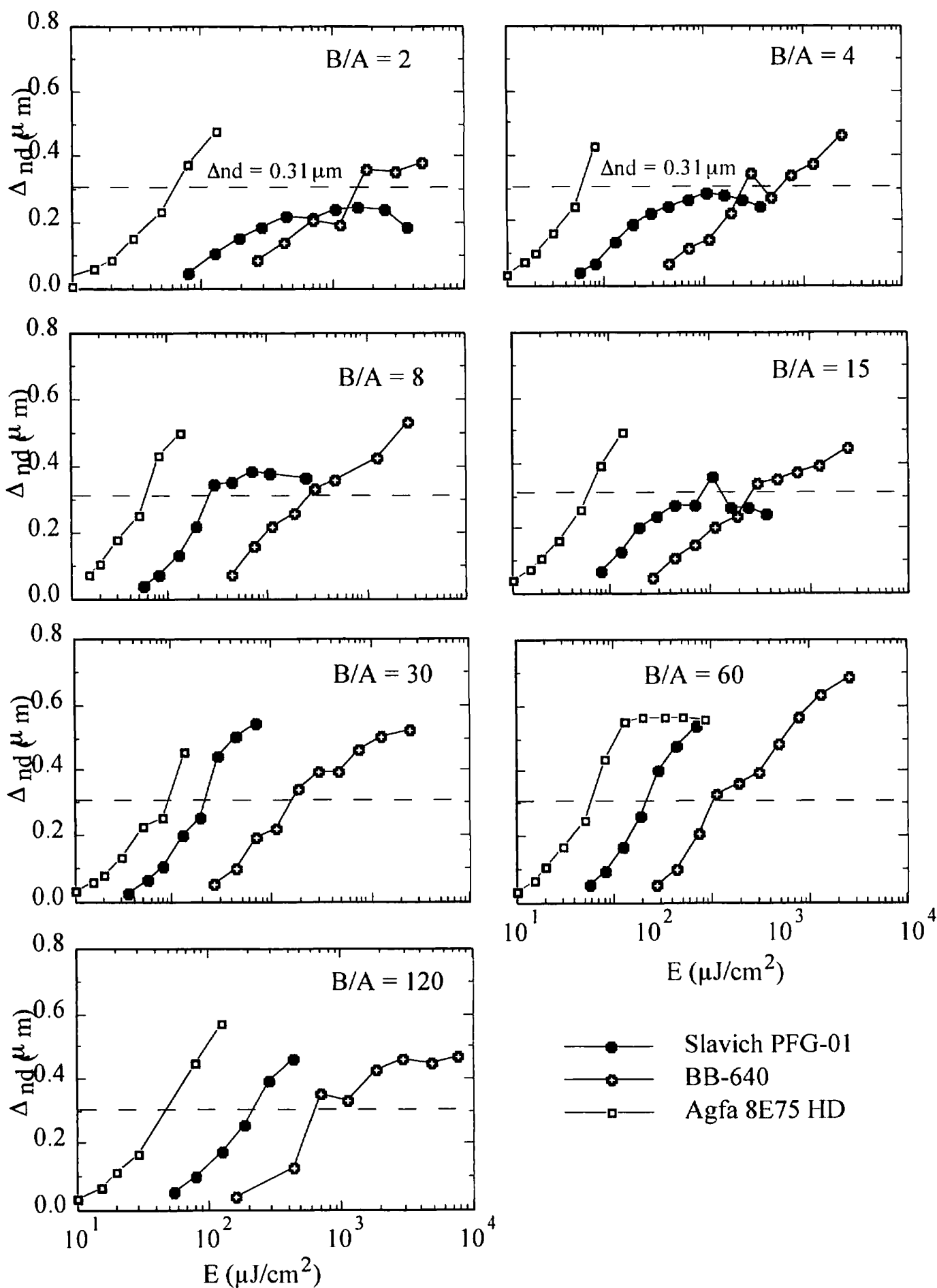

FIGURE 3

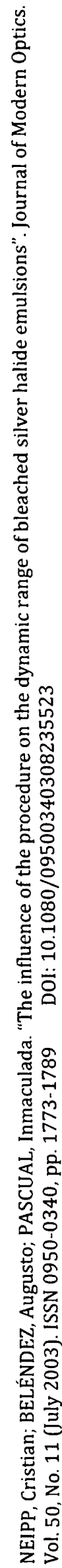




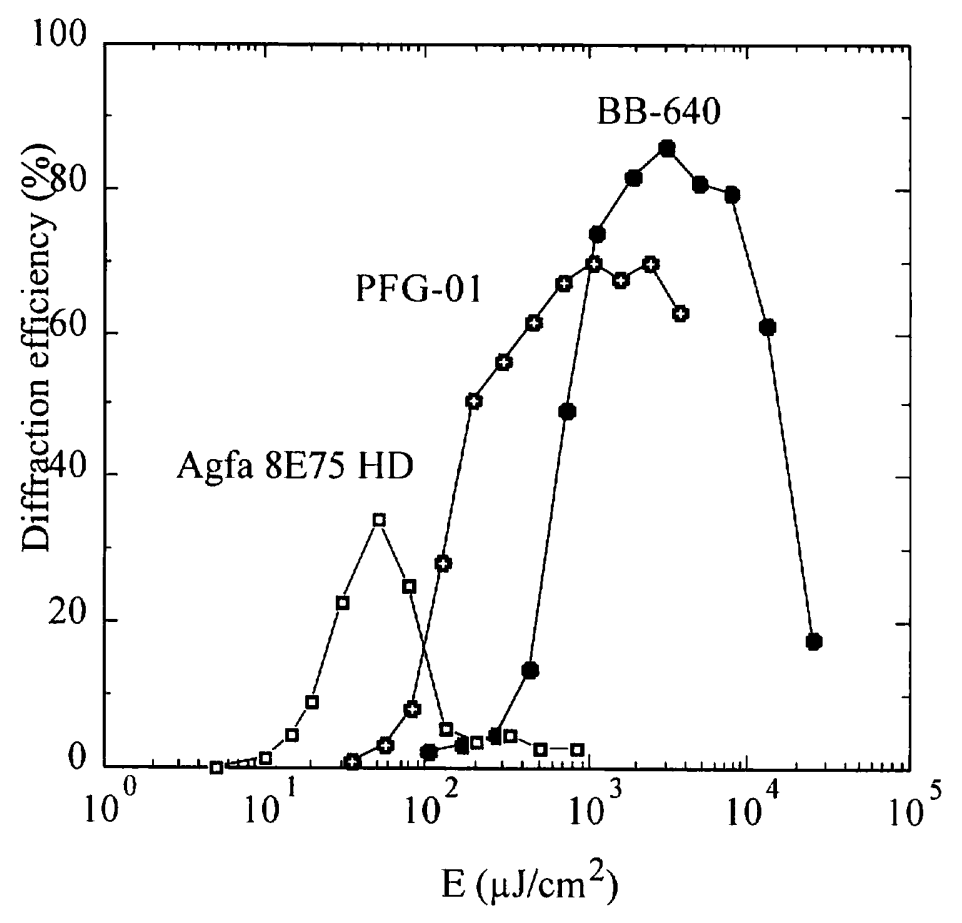

FIGURE 4

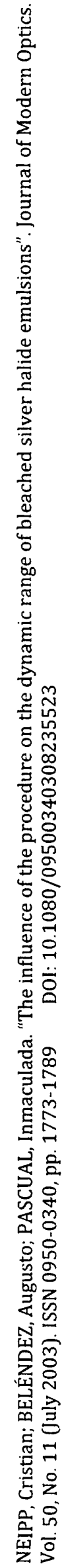




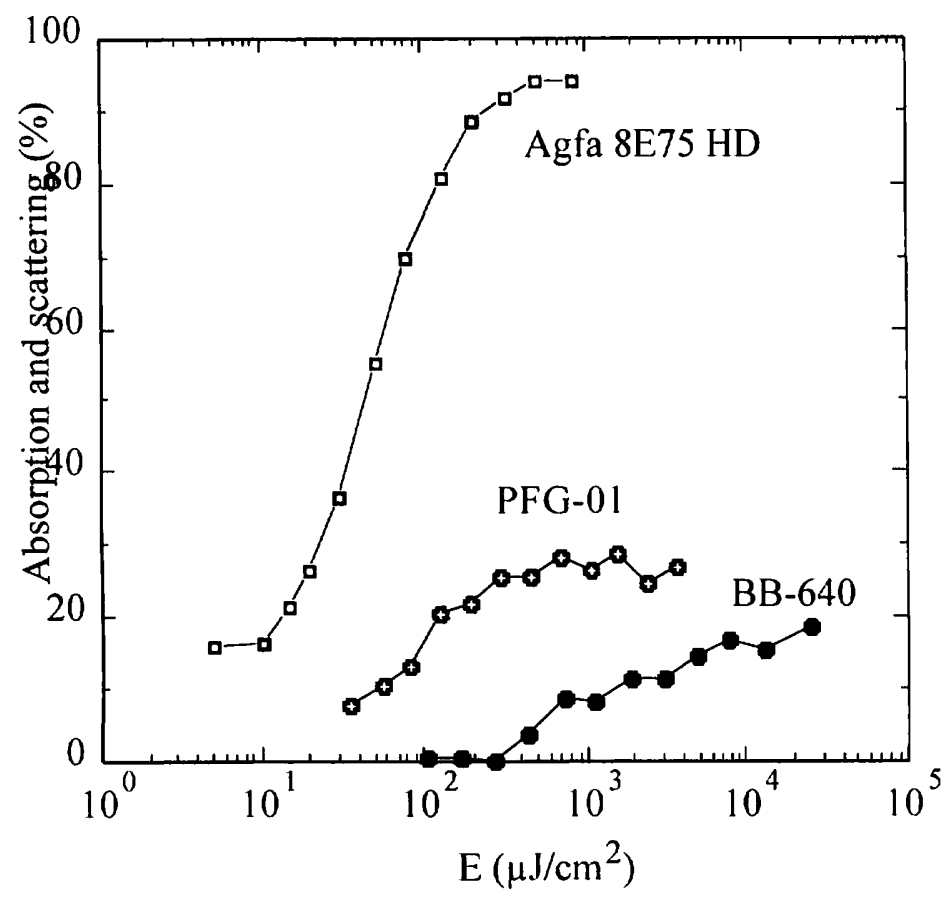

FIGURE 5

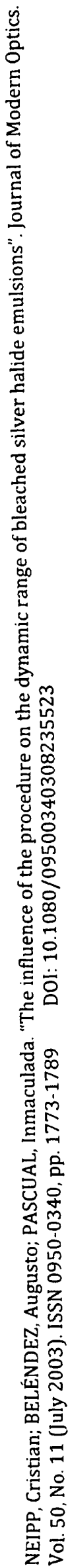




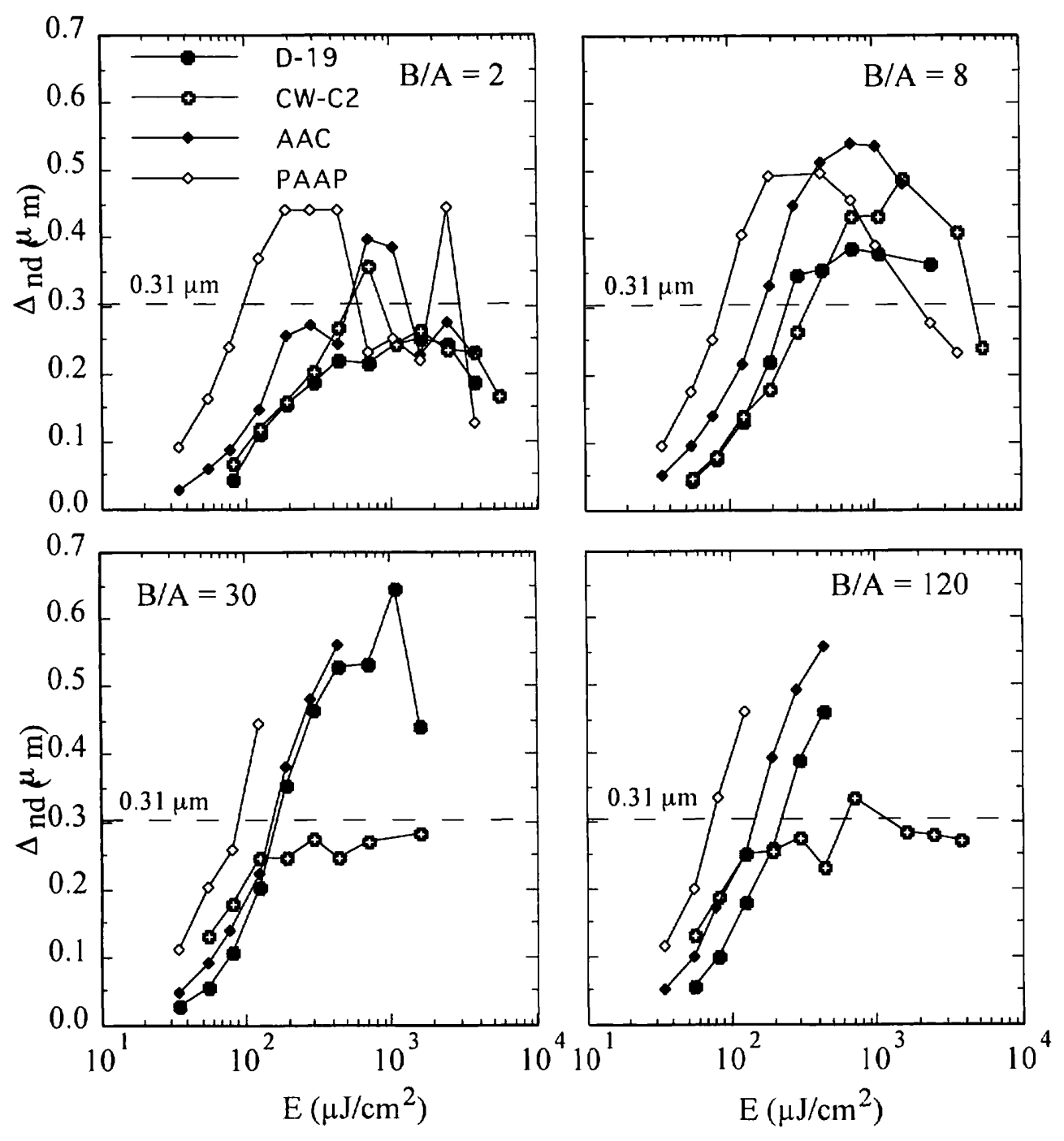

FIGURE 6

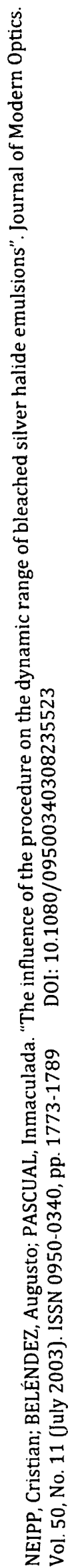



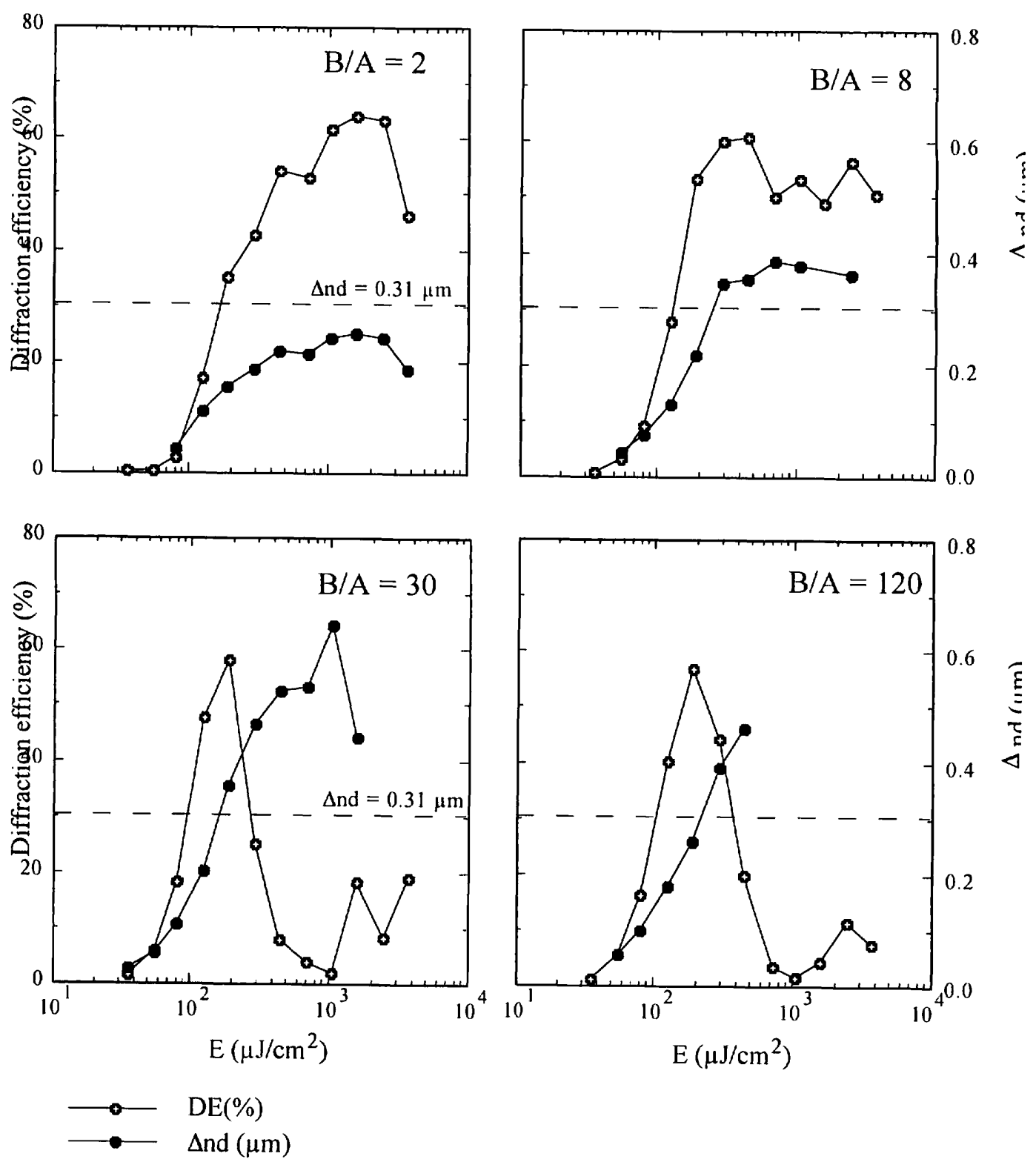

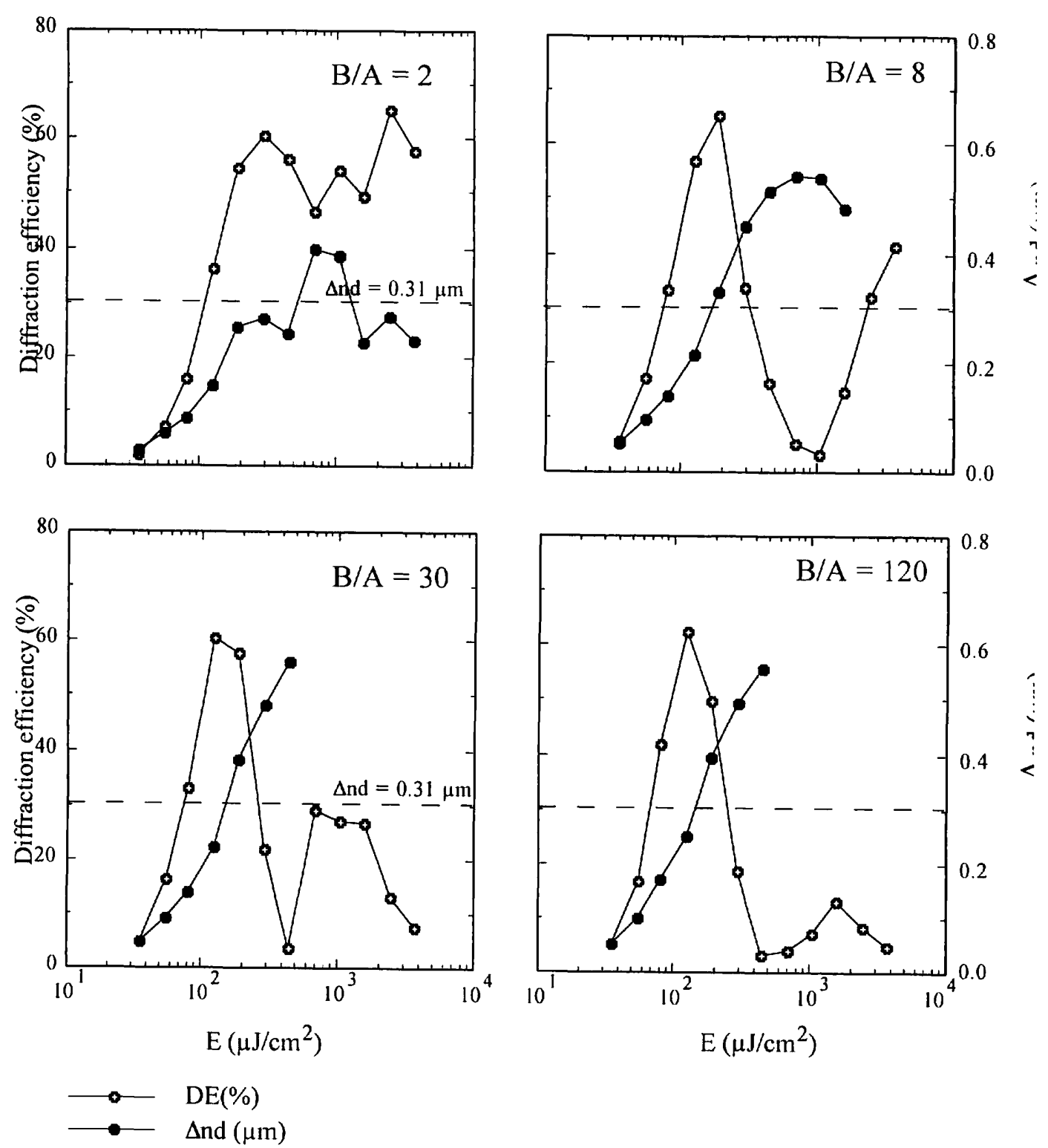

FIGURE 8 

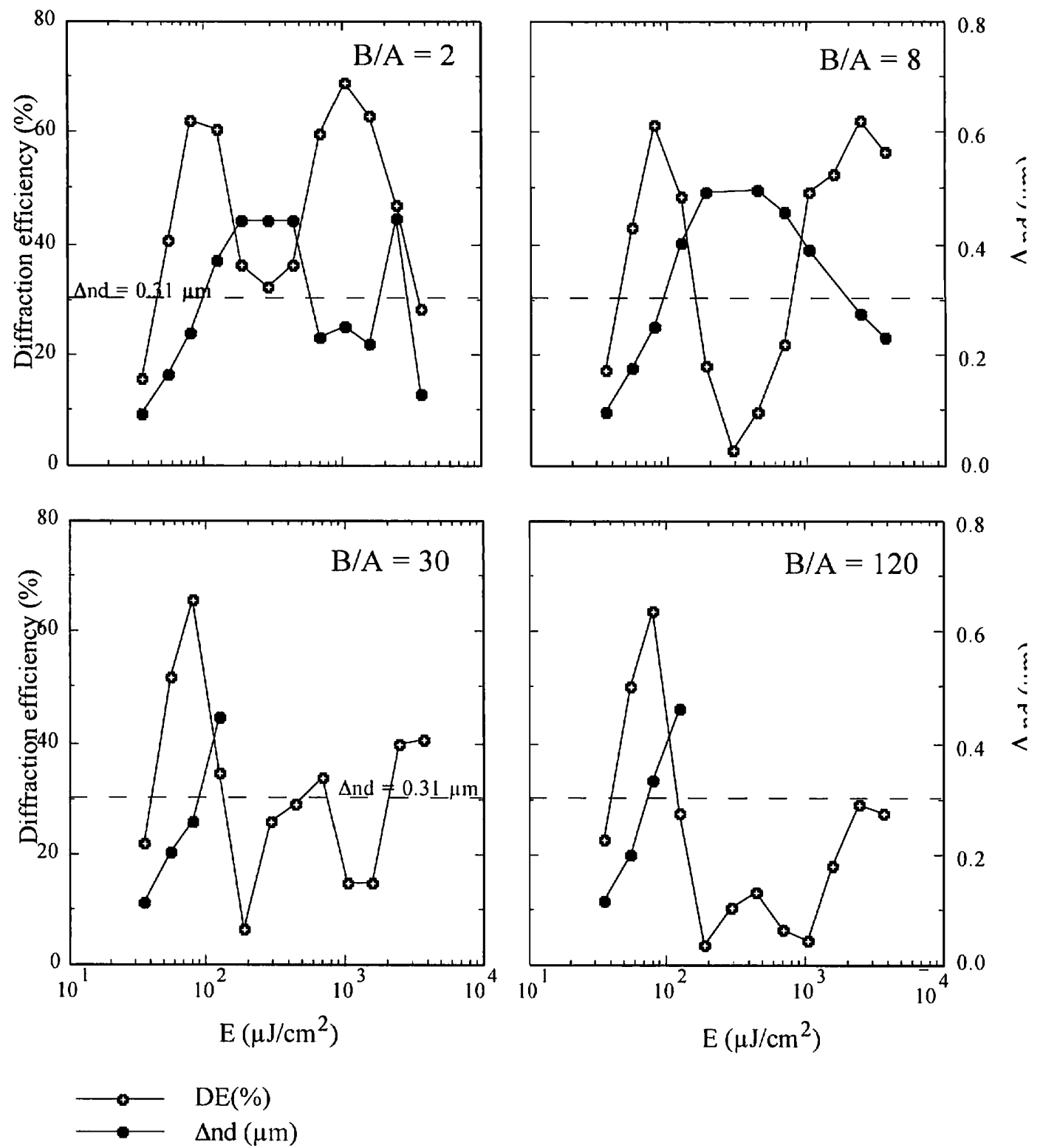

FIGURE 9 

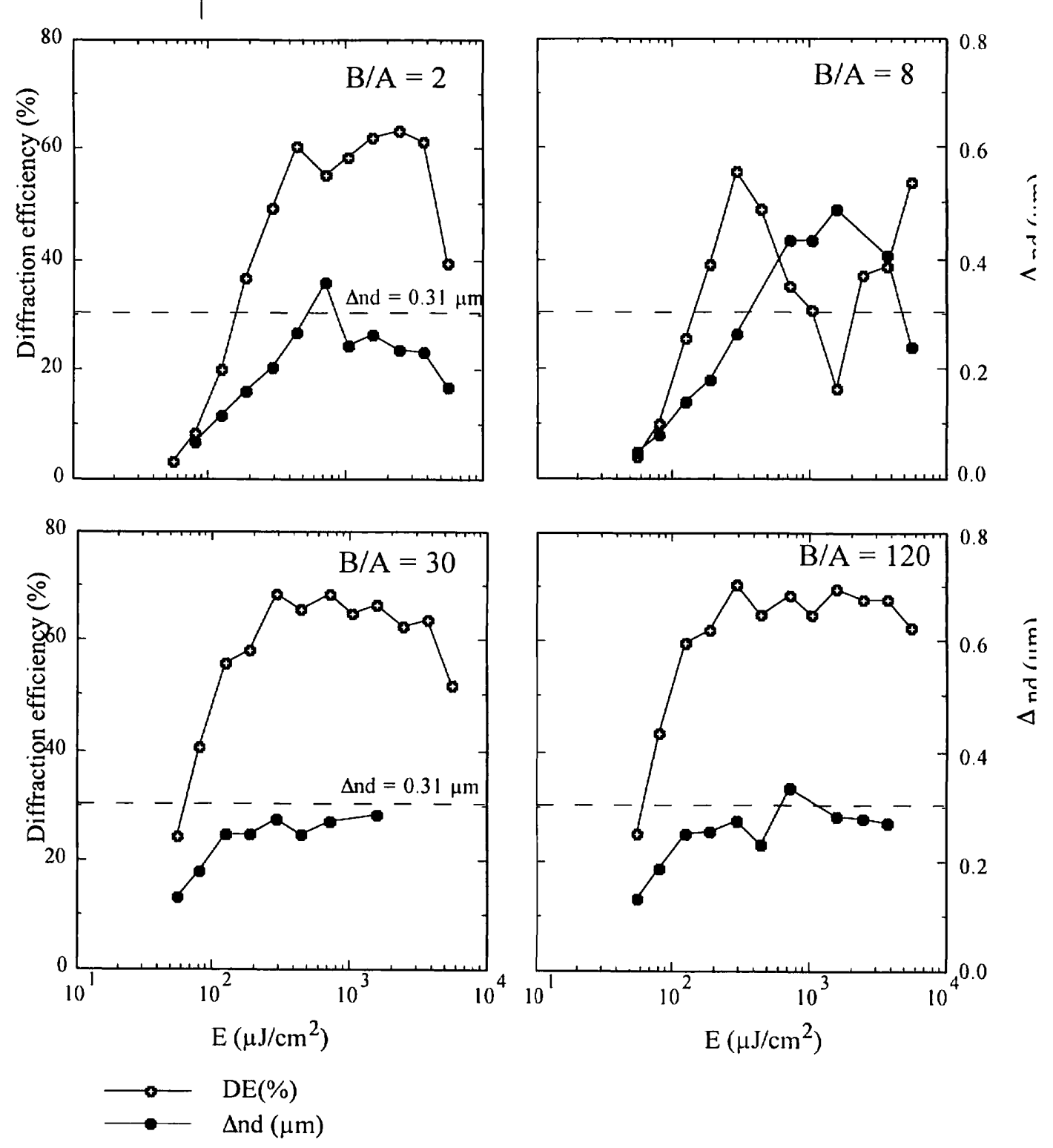

FIGURE 10 


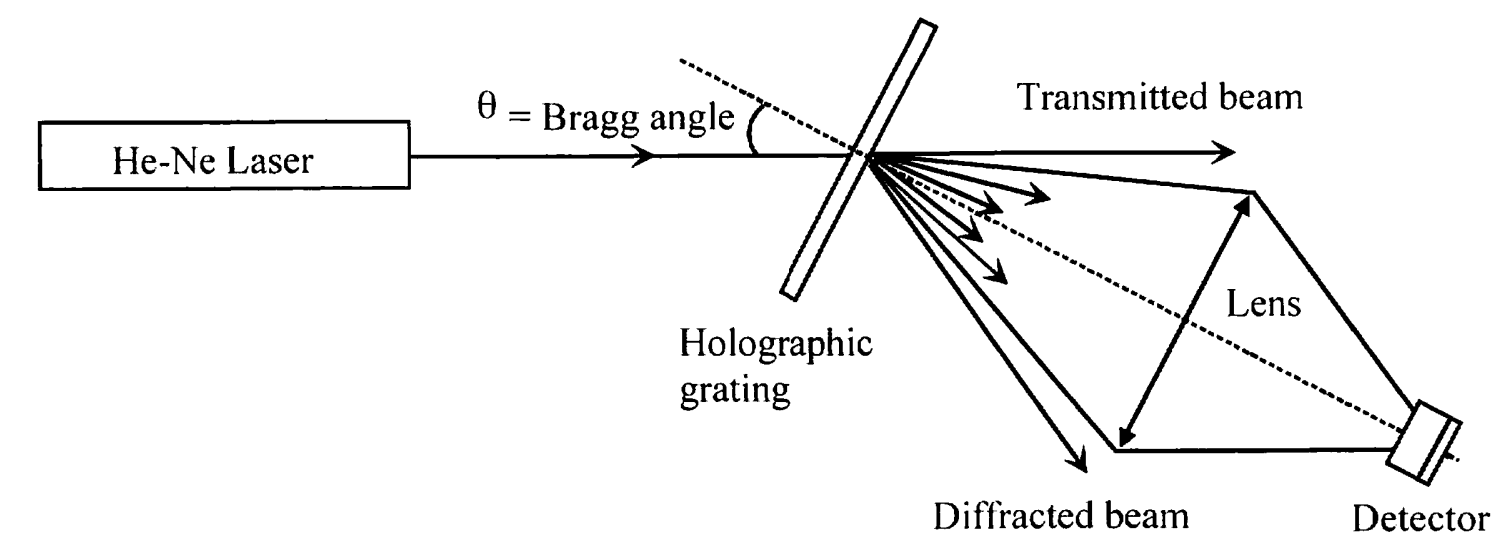

FIGURE 11 


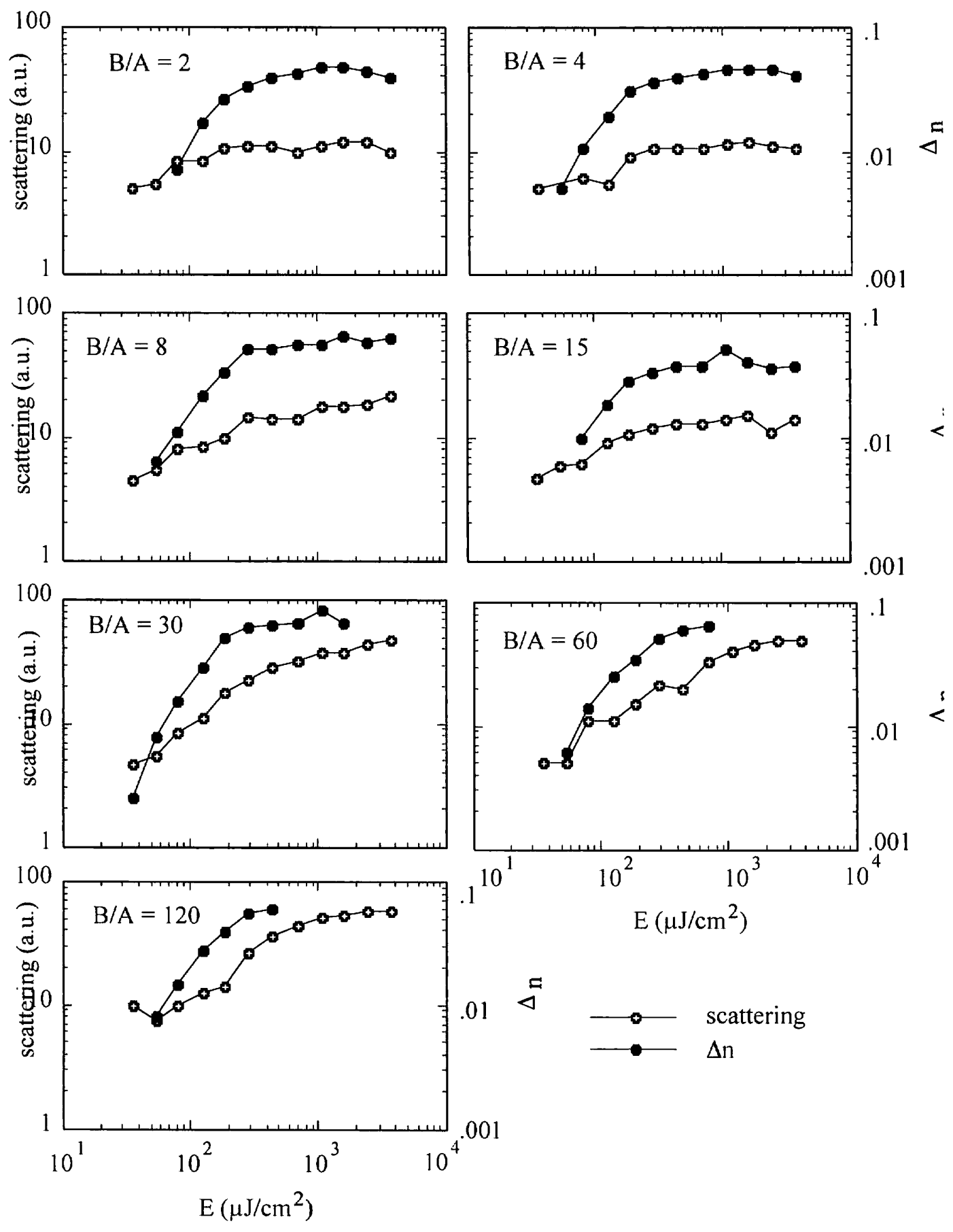

FIGURE 12

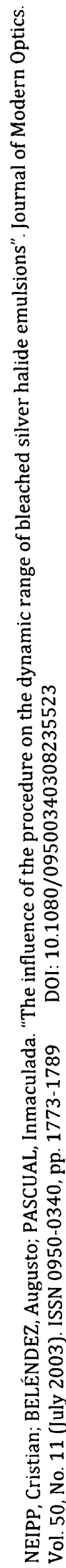

